\title{
Synthesis and Characterization of Some Quinazoline Derivatives as Potential Antimicrobial Agents under Microwave Irradiation
}

\author{
Sarika Mehta, Neelam Swarnkar, Madhuri Vyas, Jitendra Vardia, Pinki B. Punjabi, and Suresh C. Ameta \\ Microwave Chemistry Laboratory, Department of Chemistry, University College of Science, ML Sukhadia University, \\ Udaipur-313001(Raj), INDIA. "E-mail: ameta_sc(ayahoo.com \\ Received Angust 8, 2007
}

\begin{abstract}
Under the framework of green chemistry, an efficient and extremely fast procedure for the synthesis of 5a-h through four-step procedure starting from 2-arylidenetetralin-1-one 1a-d under microwave irradiation is described. $\Lambda$ considerable increase in the reaction rate has been observed with better yield. The structures of the synthesized compounds have been characterized on the basis of their elemental analysis and spectral data. Synthesized compounds $\mathbf{5 a - h}$ was evaluated for their antimicrobial activity. Some of the compounds exhibited appreciable activity.
\end{abstract}

Key Words : Tetralone, Chalcone, Thiazolidinone, Isoxalzoline, Microwave irradiation

\section{Introduction}

In recent years thiazolidinone derivatives have gained unique importance due to broad spectrum of pharmacological activities ${ }^{1,2}$ which are reflected by their use as analgesic, ${ }^{3}$ antifungal, ${ }^{4}$ antiinflamatory ${ }^{5}{ }^{6}$ and anti HIV activity. ${ }^{7}$ Some of thiazolidinone derivatives exhibited resistance against some potentially fatal disease such as cancer, ${ }^{8.9}$ tuberculosis ${ }^{10}$ etc. The importance of fused isoxazole, common sources for the development of new therapeutic agents, ${ }^{I I, 12}$ is well known. Quinazoline is a big family of heterocyclic compounds which has shown broad variety of biological activity profiles, ${ }^{13,14}$ such as analgesic, narcotic, diuretic, antihypertensive, antimalarial, sedative, hypoglycemic, antibiotic, antitumoral and many others. These examples clearly demonstrate the remarkable potential of quinazoline derivatives as a source of useful pharmacophore for new drug evolution. As our interest in "search for bioactive heterocycles," we sought an unexplored, synthetically accessible heterocyclic template (quinazoline) capable of bearing some potential pharmacophores to elicit and enhance the inherent biological activity. Thus either synthesis of this heterocyclic nucleus or its incorporation into some established pharmacophores like thiazolidinone $e^{15}$ and isoxazole, ${ }^{16}$ is of continuing interest and has been the aim of the present investigation. In view of the high biological activity of compounds related to quinazoline in particular and thiazolidinone and isoxazole in general we became interested in devising eco-friendly synthetic methodologies for the preparation of large quantities for pharmacological evolution and other purposes. Microwave induced organic transformations ${ }^{17.18}$ stands among the altemative routes proposed during the last decade or so due to various reasons like higher yields in shortest possible time and above all ecofriendliness. This paper describes an efficient and operationally simple method for the synthesis of title compounds under microwave irradiation versus conventional thermal cyclisation for comparative purpose.

\section{Results and Discussion}

The high throughput afforded by microwave technology is exemplified by the successful synthesis of the targeted compounds by the installation of various moieties in one molecule through a multistep reaction procedure. In order to achieve our objective 2-arylidenetetralin-1-ones 1a-d, obtained by the condensation of $\alpha$-tetralone with appropriate aromatic aldehydes, were treated with thiourea in alkaline medium yielded 4-aryl-3,4,5,6-tetralhydrobenzo[h]quinazolin-2(1H)-thiones $\mathbf{2 a - d}$ in good yield. Compound $\mathbf{2 a}$ showed characteristic IR absorption band in the regions $3333 \mathrm{~cm}^{-1}$ $(\mathrm{N}-\mathrm{H}), 1220 \mathrm{~cm}^{-1}(\mathrm{C}=\mathrm{S})$ and $750 \mathrm{~cm}^{-1}$ (C-Cl). Its ${ }^{1} \mathrm{H} \mathrm{NMR}$ spectrum exhibited two singlet at $\delta 5.05\left(-\mathrm{CH}_{0}\right)$ and $\delta 6.40$ $(\mathrm{N}-\mathrm{H})$. Furthermore it showed two characteristic quinazoline triplets $(J=8.0 \mathrm{~Hz})$ at $\delta 2.60$ and at $\delta 2.15$. The thiones $\mathbf{2 a - d}$ on reaction with chloroacetic acid and subsequent cyclisation of the intermediates in situ was likely to give 5-aryl-2, 3,6,7-tetrahydro-3-oxo-5H-thiazolo[2,3-b]benzo[h]quinazolines 3a-d. IR spectrum of compound $\mathbf{3 a}$ was in well agreement to the assigned structure showing the absence of band at $3333 \mathrm{~cm}^{-1}$ of N-H str. and at $1220 \mathrm{~cm}^{-1}$ of $(\mathrm{C}=\mathrm{S})$ and showing the band at $1710 \mathrm{~cm}^{-1}$ ( $\mathrm{C}=\mathrm{O}$ thiazolidinone ring) and $701 \mathrm{~cm}^{-1}$ (C-S-C). Another possible isomeric structure 6a-d could be proposed for the prepared compounds. However the structures 6a-d can be readily discarded on the basis of the fact that in benzoquinazoline $\mathbf{2 a - d}$, the most nucleophile site is the cyclic secondary ' $N$ ' atom at position3. Expansion of the 'H NMR spectrum further confirmed the structure of these compounds that gave an interesting feature of $\mathrm{H}_{a}$ proton. The $\mathrm{H}_{a}$ proton in 2 a resonated at $\delta 5.05$ where as cyclic product obtained from $\mathbf{2 a}$ gave the signal for same $\mathrm{H}_{\mathfrak{a}}$ proton at $\delta 5.70$. It confirms the proposed structure $\mathbf{3 a}$ in which the $\mathrm{H}_{a}$ proton was de-shielded by the carbonyl group of the thiazolidinone ring and appeared downfield as compared to the same proton in $\mathbf{2 a}$. Thiazolidinone derivatives 3a-d was condensed with aromatic aldehydes to give arylidene derivatives $\mathbf{4 a - h}$ which were characterized by IR and 
'H NMR spectral data. The IR spectrum of compounds 4a showed the absence of carbonyl absorption of thiazolidinone at $1710 \mathrm{~cm}^{-1}$ and the presence of chalcone carbonyl, band $(\mathrm{C}=\mathrm{C}-\mathrm{C}=\mathrm{O})$ at $1670 \mathrm{~cm}^{-1}$. Its ${ }^{1} \mathrm{H}$ NMR spectrum displayed new singlet at $\delta 6.60$ attribute to chalcone moiety $\left(=\mathrm{CH}_{\mathrm{b}}\right.$ proton). The arylidene compounds $\mathbf{4 a - h}$ on reaction with hydroxylamine hydrochloride afforded their respective in situ oxidized products 5a-h. The disappearance of the carbonyl str. at $1670 \mathrm{~cm}^{-1}$ and appearance of band at 1075 $\mathrm{cm}^{-1}(\mathrm{C}-\mathrm{O})$ and $900 \mathrm{~cm}^{-1}(\mathrm{~N}-\mathrm{O})$ in $\mathrm{R}$ spectrum for 5a confirms the installation of isoxazole moieties in $4 a$. The substrate 4 having $\mathrm{a}-\mathrm{C}=\mathrm{C}-\mathrm{C}=\mathrm{O}-(\alpha-, \beta$-unsaturated carbon-

Table 1. Characterization data of synthesized compounds

\begin{tabular}{|c|c|c|c|c|c|c|c|c|}
\hline \multirow{2}{*}{ Compd. } & \multirow{2}{*}{ m.p. $(C)$} & \multicolumn{2}{|c|}{ Yield $(\%)$} & \multirow{2}{*}{$\begin{array}{l}\text { Molecular Formula } \\
\text { (M.W.) }\end{array}$} & \multicolumn{4}{|c|}{ Calculated/Found $(\%)$} \\
\hline & & Classical & Microwave & & $\mathrm{C}$ & $\mathrm{H}$ & $\mathrm{N}$ & $S$ \\
\hline \multirow[t]{2}{*}{$2 a$} & 210 & 63 & 85 & $\mathrm{C}_{18} \mathrm{H}_{15} \mathrm{~N}_{2} \mathrm{SCl}$ & 66.25 & 4.60 & 8.58 & 9.81 \\
\hline & $(\mathrm{EtOH})$ & & & $(326)$ & 65.92 & 4.49 & 8.52 & 9.77 \\
\hline \multirow[t]{2}{*}{$2 \mathbf{b}$} & 185 & 55 & 90 & $\mathrm{C}_{18} \mathrm{H}_{15} \mathrm{~N}_{2} \mathrm{SF}$ & 69.67 & 4.83 & 9.03 & 10.32 \\
\hline & $(\mathrm{EtOH})$ & & & $(310)$ & 69.40 & 4.14 & 9.02 & 10.29 \\
\hline \multirow[t]{2}{*}{$2 \mathrm{c}$} & 156 & 52 & 75 & $\mathrm{C}_{19} \mathrm{H}_{18} \mathrm{~N}_{2} \mathrm{SO}$ & 70.80 & 5.59 & 8.69 & 9.93 \\
\hline & $(\mathrm{EtOH})$ & & & (322) & 70.52 & 5.10 & 8.64 & 9.88 \\
\hline \multirow[t]{2}{*}{$2 d$} & 225 & 51 & 67 & $\mathrm{C}_{18} \mathrm{H}_{16} \mathrm{~N}_{2} \mathrm{SCl}$ & 73.97 & 5.47 & 9.58 & 10.95 \\
\hline & $(\mathrm{EtOH})$ & & & $(292)$ & 73.67 & 5.30 & 9.52 & 10.90 \\
\hline \multirow[t]{2}{*}{$3 a$} & 175 & 45 & 86 & $\mathrm{C}_{20} \mathrm{H}_{15} \mathrm{~N}_{2} \mathrm{SOCl}$ & 65.57 & 4.09 & 7.65 & 8.74 \\
\hline & $(\mathrm{EtOH})$ & & & $(366)$ & 65.64 & 3.84 & 7.63 & 8.77 \\
\hline \multirow[t]{2}{*}{$\mathbf{3 b}$} & 190 & 63 & 82 & $\mathrm{C}_{20} \mathrm{H}_{15} \mathrm{~N}_{2} \mathrm{SOF}$ & 68.57 & 4.28 & 8.00 & 9.14 \\
\hline & $(\mathrm{EtOH})$ & & & $(350$ & 68.42 & 4.02 & 8.00 & 9.10 \\
\hline \multirow[t]{2}{*}{$3 c$} & 238 & 58 & 83 & $\mathrm{C}_{21} \mathrm{H}_{18} \mathrm{~N}_{2} \mathrm{SO}_{2}$ & 69.61 & 5.24 & 7.73 & 8.83 \\
\hline & $(\mathrm{EtOH})$ & & & $(362)$ & 69.76 & 4.72 & 7.71 & 8.77 \\
\hline \multirow[t]{2}{*}{$3 d$} & 184 & 50 & 79 & $\mathrm{C}_{20} \mathrm{H}_{15} \mathrm{~N}_{2} \mathrm{SO}$ & 72.28 & 4.51 & 8.43 & 9.63 \\
\hline & $(\mathrm{EtOH})$ & & & (331) & 72.26 & 4.44 & 8.40 & 9.62 \\
\hline \multirow[t]{2}{*}{$4 a$} & 178 & 61 & 81 & $\mathrm{C}_{27} \mathrm{H}_{18} \mathrm{~N}_{2} \mathrm{SOCl}_{2}$ & 66.25 & 3.68 & 5.72 & 6.54 \\
\hline & $\left(\mathrm{CHCl}_{3}\right)$ & & & (489) & 66.20 & 3.29 & 5.71 & 6.52 \\
\hline \multirow[t]{2}{*}{$4 b$} & 245 & 57 & 91 & $\mathrm{C}_{27} \mathrm{H}_{18} \mathrm{~N}_{2} \mathrm{SOCIF}$ & 68.64 & 3.81 & 5.93 & 6.77 \\
\hline & $\left(\mathrm{CHCl}_{3}\right)$ & & & $(472)$ & 68.21 & 3.39 & 5.91 & 6.72 \\
\hline \multirow[t]{2}{*}{$4 c$} & 215 & 54 & 88 & $\mathrm{C}_{28} \mathrm{H}_{21} \mathrm{~N}_{2} \mathrm{SO}_{2} \mathrm{Cl}$ & 69.42 & 3.71 & 5.78 & 6.61 \\
\hline & $\left(\mathrm{CHCl}_{3}\right)$ & & & (484) & 69.31 & 3.68 & 5.71 & 6.53 \\
\hline \multirow[t]{2}{*}{$4 \mathbf{d}$} & 280 & 49 & 79 & $\mathrm{C}_{27} \mathrm{H}_{19} \mathrm{~N}_{2} \mathrm{SOCl}$ & 74.00 & 4.18 & 6.16 & 7.04 \\
\hline & $\left(\mathrm{CHCl}_{3}\right)$ & & & $(454)$ & 71.60 & 3.76 & 6.13 & 7.02 \\
\hline \multirow[t]{2}{*}{$4 e$} & 165 & 52 & 83 & $\mathrm{C}_{27} \mathrm{H}_{18} \mathrm{~N}_{2} \mathrm{SOCIF}$ & 68.64 & 3.81 & 5.93 & 6.77 \\
\hline & $\left(\mathrm{CHCl}_{3}\right)$ & & & $(472)$ & 68.55 & 3.40 & 5.90 & 6.70 \\
\hline \multirow[t]{2}{*}{$4 f$} & 260 & 62 & 85 & $\mathrm{C}_{27} \mathrm{H}_{18} \mathrm{~N}_{2} \mathrm{SOF}_{2}$ & 71.05 & 3.94 & 6.14 & 7.01 \\
\hline & $\left(\mathrm{CHCl}_{3}\right)$ & & & $(456)$ & 71.03 & 3.54 & 6.14 & 7.00 \\
\hline \multirow[t]{2}{*}{$4 \mathrm{~g}$} & 148 & 56 & 89 & $\mathrm{C}_{28} \mathrm{H}_{21} \mathrm{~N}_{2} \mathrm{SO}_{2} \mathrm{~F}$ & 71.79 & 4.48 & 5.98 & 6.83 \\
\hline & $\left(\mathrm{CHCl}_{3}\right)$ & & & $(468)$ & 71.06 & 4.05 & 5.93 & 6.80 \\
\hline $4 h$ & 213 & 61 & 84 & $\mathrm{C}_{27} \mathrm{H}_{19} \mathrm{~N}_{2} \mathrm{SOF}$ & 73.97 & 4.10 & 6.39 & 7.30 \\
\hline & $\left(\mathrm{CHCl}_{3}\right)$ & & & $(438)$ & 73.26 & 3.91 & 6.38 & 7.20 \\
\hline $5 a$ & 225 & 50 & 90 & $\mathrm{C}_{27} \mathrm{H}_{19} \mathrm{~N}_{3} \mathrm{SOCl}_{2}$ & 64.28 & 3.76 & 8.33 & 6.34 \\
\hline & $(\mathrm{EtOH})$ & & & (504) & 64.20 & 3.20 & 8.30 & 6.31 \\
\hline $\mathbf{5 b}$ & 210 & 45 & 88 & $\mathrm{C}_{27} \mathrm{H}_{19} \mathrm{~N}_{3} \mathrm{SOCJF}$ & 66.52 & 3.90 & 8.62 & 6.57 \\
\hline & $(\mathrm{EtOH})$ & & & (487) & 66.50 & 3.30 & 8.64 & 6.52 \\
\hline $5 c$ & 192 & 60 & 85 & $\mathrm{C}_{28} \mathrm{H}_{22} \mathrm{~N}_{3} \mathrm{SO}_{2} \mathrm{Cl}$ & 67.33 & 4.40 & 8.41 & 6.41 \\
\hline & $(\mathrm{EtOH})$ & & & $(499)$ & 66.60 & 3.83 & 8.12 & 6.32 \\
\hline 5d & 205 & 51 & 87 & $\mathrm{C}_{27} \mathrm{H}_{26} \mathrm{~N}_{3} \mathrm{SOCl}$ & 69.08 & 4.26 & 8.95 & 6.82 \\
\hline & $(\mathrm{EtOH})$ & & & $(469)$ & 69.00 & 3.63 & 8.93 & 6.81 \\
\hline $5 e$ & 188 & 54 & 92 & $\mathrm{C}_{27} \mathrm{H}_{19} \mathrm{~N}_{3} \mathrm{SOCIF}$ & 66.52 & 3.90 & 8.62 & 6.57 \\
\hline & $(\mathrm{EtOH})$ & & & $(487)$ & 66.40 & 3.29 & 8.62 & 6.54 \\
\hline $5 f$ & 218 & 52 & 88 & $\mathrm{C}_{27} \mathrm{H}_{19} \mathrm{~N}_{3} \mathrm{SOF}_{2}$ & 68.78 & 4.03 & 8.91 & 6.79 \\
\hline & $(\mathrm{EtOH})$ & & & (471) & 68.20 & 3.41 & 8.90 & 6.72 \\
\hline $5 g$ & 220 & 60 & 89 & $\mathrm{C}_{28} \mathrm{H}_{22} \mathrm{~N}_{3} \mathrm{SO}_{2} \mathrm{~F}$ & 69.56 & 4.55 & 8.69 & 6.62 \\
\hline & $(\mathrm{EtOH})$ & & & (483) & 69.50 & 3.97 & 8.60 & 6.60 \\
\hline $5 \mathrm{~h}$ & 198 & 55 & 90 & $\mathrm{C}_{27} \mathrm{H}_{20} \mathrm{~N}_{3} \mathrm{SOF}$ & 71.52 & 4.41 & 9.27 & 7.06 \\
\hline & $(\mathrm{EtOH})$ & & & $(453)$ & 71.40 & 3.77 & 9.20 & 7.01 \\
\hline
\end{tabular}


Table 2. Spectral data of synthesized compounds

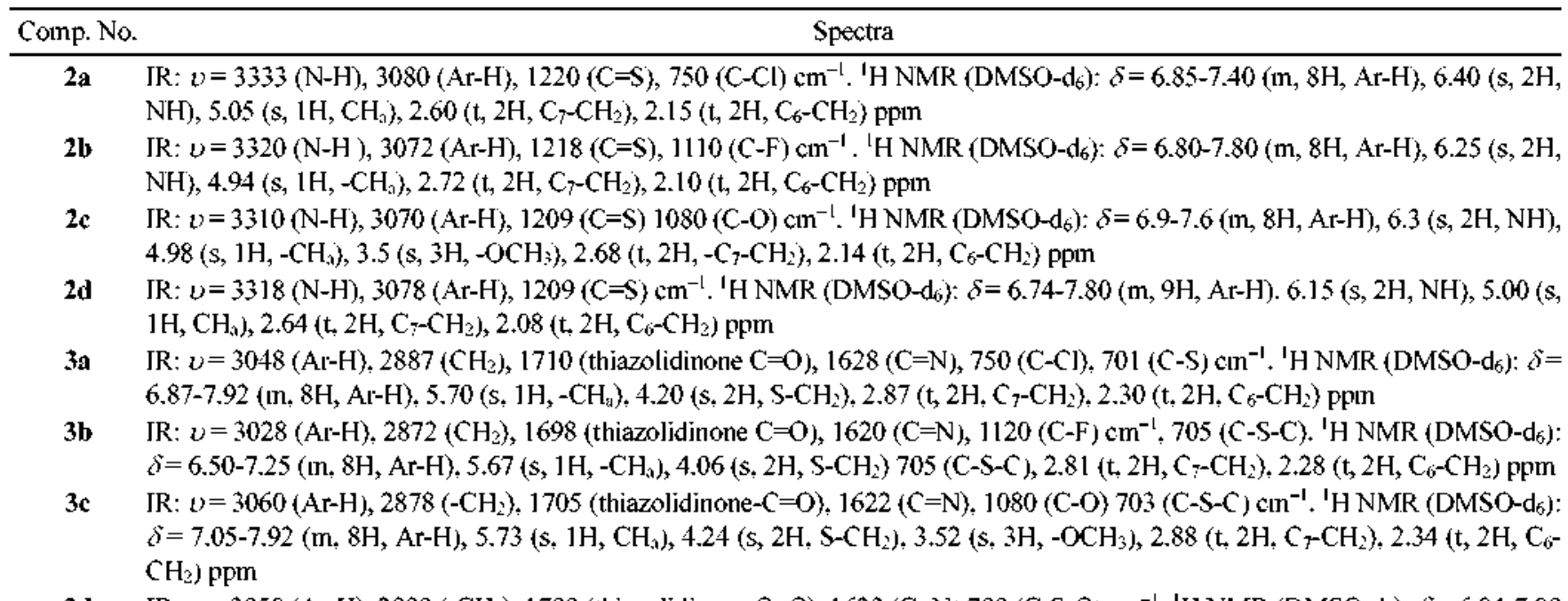

3d IR: $v=3050(\mathrm{Al}-\mathrm{H}), 2890\left(-\mathrm{CH}_{2}\right), 1700$ (thiazolidinone $\left.\mathrm{C}=0\right), 1623(\mathrm{C}=\mathrm{N}) 700(\mathrm{C}-\mathrm{S}-\mathrm{C}) \mathrm{cm}^{-1} .{ }^{\prime} \mathrm{H}$ NMR $\left(\mathrm{DMSO}-\mathrm{d}_{6}\right): \delta=6.84-7.90$ (in, 9H, Ar-H), $5.68\left(\mathrm{~s}, 1 \mathrm{H}, \mathrm{CH}_{3}\right), 4.09\left(\mathrm{~s}, 2 \mathrm{H}, \mathrm{S}-\mathrm{CH}_{2}\right), 2.77\left(\mathrm{t}, 2 \mathrm{H}, \mathrm{C}_{7}-\mathrm{CH}_{2}\right), 2.27\left(\mathrm{t}, 2 \mathrm{H}, \mathrm{C}_{6}-\mathrm{CH}_{2}\right) \mathrm{ppm}$

$4 a$ IR: $v=3100(\mathrm{Al}-\mathrm{H}), 1670(\mathrm{C}=\mathrm{O}), 1630(\mathrm{C}=\mathrm{N}), 748(\mathrm{C}-\mathrm{Cl}), 686(\mathrm{C}-\mathrm{S}-\mathrm{C}) \mathrm{cm}^{-1}$. ' $\mathrm{H}$ NMR (DMSO-d $) ; \delta=6.90-7.85(\mathrm{~m}, 12 \mathrm{H}, \mathrm{Al}-$ $\mathrm{H}), 6.60\left(\mathrm{~s}, 1 \mathrm{H}, \mathrm{CH}_{\mathrm{b}}\right), 5.70\left(\mathrm{~s}, 1 \mathrm{H}, \mathrm{CH}_{3}\right), 2.70\left(\mathrm{t}, 2 \mathrm{H}, \mathrm{C}_{7}-\mathrm{CH}_{2}\right), 2.10\left(\mathrm{t}, 2 \mathrm{H}, \mathrm{C}_{6}-\mathrm{CH}_{2}\right) \mathrm{ppm}$

4b IR: $v=3078(\mathrm{Ar}-\mathrm{H}), 1668(\mathrm{C}=0), 1625(\mathrm{C}=\mathrm{N}), 1180(\mathrm{C}-\mathrm{F}), 745(\mathrm{C}-\mathrm{Cl}), 684(\mathrm{C}-\mathrm{S}-\mathrm{C}), \mathrm{cm}^{-1}$. 'H NMR $\left(\mathrm{DMSO}-\mathrm{d}_{6}\right): \delta=6.90-7.85$ $(\mathrm{m}, 12 \mathrm{H}, \mathrm{Ar}-\mathrm{H}), 6.52\left(\mathrm{~s}, 1 \mathrm{H}, \mathrm{CH}_{\mathrm{b}}\right), 5.68\left(\mathrm{~s}, 1 \mathrm{H}, \mathrm{CH}_{3}\right), 2.65\left(\mathrm{t}, 2 \mathrm{H}, \mathrm{C}_{7}-\mathrm{CH}_{2}\right), 2.08\left(\mathrm{t}, 2 \mathrm{H}, \mathrm{C}_{6}-\mathrm{CH}_{2}\right) \mathrm{ppm}$

$4 \mathrm{IR}: \nu=3050(\mathrm{Ar}-\mathrm{H}), 1665(\mathrm{C}=\mathrm{N}), 1075(\mathrm{C}=\mathrm{O}), 748(\mathrm{C}-\mathrm{Cl}), 680(\mathrm{C}-\mathrm{S}-\mathrm{C}) \mathrm{cm}^{-1}$. 'H NMR (DMSO-d $): \delta=6.76-7.81(\mathrm{~m}, 12 \mathrm{H}, \mathrm{Ar}-$ $\mathrm{H}), 6.50\left(\mathrm{~s}, 1 \mathrm{H}, \mathrm{CH}_{\mathrm{b}}\right), 5.59\left(\mathrm{~s}, 1 \mathrm{H}, \mathrm{CH}_{3}\right), 3.82\left(\mathrm{~s}, 3 \mathrm{H}, \mathrm{OCH}_{3}\right), 2.64\left(\mathrm{t}, 2 \mathrm{H}, \mathrm{C}_{7}-\mathrm{CH}_{2}\right), 2.09\left(\mathrm{t}, 2 \mathrm{H}, \mathrm{C}_{6}-\mathrm{CH}_{2}\right) \mathrm{ppm}$

4d IR: $v=3072(\mathrm{Ar}-\mathrm{H}), 1662(\mathrm{C}=\mathrm{O}), 1620(\mathrm{C}=\mathrm{N}), 745(\mathrm{C}-\mathrm{Cl}), 678(\mathrm{C}-\mathrm{S}-\mathrm{C}) \mathrm{cm}^{-1}$. 'H NMR (DMSO-d $): \delta=6.71-7.50(\mathrm{~m}, 12 \mathrm{H}, \mathrm{Ar}-$ $\mathrm{H}), 6.42\left(\mathrm{~s}, 1 \mathrm{H}, \mathrm{CH}_{\mathrm{b}}\right), 5.51\left(\mathrm{~s}, 1 \mathrm{H}, \mathrm{CH}_{3}\right), 2.60\left(\mathrm{t}, 2 \mathrm{H}, \mathrm{C}_{7}-\mathrm{CH}_{2}\right), 2.07\left(\mathrm{t}, 2 \mathrm{H}, \mathrm{C}_{6}-\mathrm{CH}_{2}\right) \mathrm{ppm}$

4e IR: $v=3068(\mathrm{Ar}-\mathrm{H}), 1660(\mathrm{C}=\mathrm{O}), 1622(\mathrm{C}=\mathrm{N}), 1168(\mathrm{C}-\mathrm{F}), 746(\mathrm{C}-\mathrm{Cl}), 672(\mathrm{C}-\mathrm{S}-\mathrm{C}) \mathrm{cm}^{-1}$. ${ }^{\mathrm{H}} \mathrm{H}$ NMR $(\mathrm{DMSO}-\mathrm{d} 6): \delta=6.62-7.42(\mathrm{~m}$, $12 \mathrm{H}, \mathrm{Ar}-\mathrm{H}), 6.48\left(\mathrm{~s}, 1 \mathrm{H}, \mathrm{CH}_{b}\right), 5.59\left(\mathrm{~s}, 1 \mathrm{H}, \mathrm{CH}_{3}\right), 2.63\left(\mathrm{t}, 2 \mathrm{H}, \mathrm{C}_{7}-\mathrm{CH}_{2}\right), 2.05\left(\mathrm{t}, 2 \mathrm{H}, \mathrm{C}_{6}-\mathrm{CH}_{2}\right) \mathrm{ppm}$

4 IR: $v=3075(\mathrm{Ar}-\mathrm{H}), 1675(\mathrm{C}=0), 1634(\mathrm{C}=\mathrm{N}), 1182(\mathrm{C}-\mathrm{F}), 684(\mathrm{C}-\mathrm{S}-\mathrm{C}) \mathrm{cm}^{-1} .{ }^{1} \mathrm{H}$ NMR (DMSO-d $): \delta=6.76-7.53(\mathrm{~m}, 12 \mathrm{H}, \mathrm{Ar}-$ $\mathrm{H}), 6.42\left(\mathrm{~s}, 1 \mathrm{H}, \mathrm{CH}_{\mathrm{b}}\right), 5.52\left(\mathrm{~s}, 1 \mathrm{H}, \mathrm{CH}_{3}\right), 2.51\left(\mathrm{t}, 2 \mathrm{H}, \mathrm{C}_{7}-\mathrm{CH}_{2}\right), 2.00\left(\mathrm{t}, 2 \mathrm{H}, \mathrm{C}_{6}-\mathrm{CH}_{2}\right) \mathrm{ppm}$

$4 \mathrm{~g}$ IR: $v=3080(\mathrm{Ar}-\mathrm{H}), 1670(\mathrm{C}=\mathrm{O}), 1627(\mathrm{C}=\mathrm{N}), 1180(\mathrm{C}-\mathrm{F}), 1050(\mathrm{C}-\mathrm{O}) \mathrm{cm}^{-1}$. 'H NMR (DMSO-d 6$): \bar{\delta}=6.80-7.72(\mathrm{~m}, 12 \mathrm{H}, \mathrm{Ar}-$ $\mathrm{H}), 6.56\left(\mathrm{~s}, 1 \mathrm{H}, \mathrm{CH}_{\mathrm{b}}\right), 5.72\left(\mathrm{~s}, 1 \mathrm{H}, \mathrm{CH}_{3}\right), 3.48\left(\mathrm{~s}, 3 \mathrm{H},-\mathrm{OCH}_{3}\right), 2.68\left(\mathrm{t}, 2 \mathrm{H}, \mathrm{C}_{7}-\mathrm{CH}_{2}\right), 2.12\left(\mathrm{t}, 2 \mathrm{H}, \mathrm{C}_{6}-\mathrm{CH}_{2}\right) \mathrm{ppm}$

4h IR: $v=3072(\mathrm{Ar}-\mathrm{H}), 1665(\mathrm{C}=\mathrm{O}), 1620(\mathrm{C}=\mathrm{N}), 1172(\mathrm{C}-\mathrm{F}) \mathrm{cm}^{-1}$. 'H NMR (DMSO-d $): \delta=6.80-7.94(\mathrm{~m}, 13 \mathrm{H}, \mathrm{Al}-\mathrm{H}), 6.58(\mathrm{~s}, 1 \mathrm{H}$, $\left.\mathrm{CH}_{\mathrm{b}}\right), 5.64\left(\mathrm{~s}, 1 \mathrm{H}, \mathrm{CH}_{\mathrm{a}}\right), 2.60\left(\mathrm{t}, 2 \mathrm{H}, \mathrm{C}_{-}-\mathrm{CH}_{2}\right), 2.15\left(\mathrm{t}, 2 \mathrm{H}, \mathrm{C}_{6}-\mathrm{CH}_{2}\right) \mathrm{ppm}$

5a IR: $v=3110(\mathrm{Ar}-\mathrm{H}), 1634(\mathrm{C}=\mathrm{N}), 1075(\mathrm{C}-\mathrm{O}), 900(\mathrm{~N}-\mathrm{O}), 755(\mathrm{C}-\mathrm{Cl}), 689(\mathrm{C}-\mathrm{S}-\mathrm{C}) \mathrm{cm}^{-1}$. 'H NMR (DMSO-d $): \delta=6.75-7.74(\mathrm{~m}$, $12 \mathrm{H}, \mathrm{Ar}-\mathrm{H}), 5.70\left(\mathrm{~s}, 1 \mathrm{H}, \mathrm{CH}_{3}\right), 4.60\left(\mathrm{~d}, 1 \mathrm{H}, \mathrm{CH}_{\mathrm{b}}-\mathrm{O}\right), 3.52(\mathrm{~d}, 1 \mathrm{H}, \mathrm{CHc}-\mathrm{S}), 2.88\left(\mathrm{t}, 2 \mathrm{H}, \mathrm{C}_{7}-\mathrm{CH}_{2}\right), 2.28\left(\mathrm{t}, 2 \mathrm{H}, \mathrm{C}_{7}-\mathrm{CH}_{2}\right) \mathrm{ppm} . \mathrm{Mass} \mathrm{m} /$ $z: 503,375,363,291,290,140,111$

5b IR: $v=3100(\mathrm{Ar}-\mathrm{H}), 1638(\mathrm{C}=\mathrm{N}), 1175(\mathrm{C}-\mathrm{F}), 1080(\mathrm{C}-\mathrm{O}), 902(\mathrm{~N}-\mathrm{O}), 752(\mathrm{C}-\mathrm{Cl}), 690(\mathrm{C}-\mathrm{S}-\mathrm{C}) \mathrm{cm}^{-1} .{ }^{\mathrm{H}} \mathrm{H}$ NMR (DMSO-d $): \delta=$ $6.60-7.80(\mathrm{~m}, 12 \mathrm{H}, \mathrm{Al}-\mathrm{H}), 5.68\left(\mathrm{~s}, 1 \mathrm{H}, \mathrm{CH}_{3}\right), 4.58\left(\mathrm{~d}, 1 \mathrm{H}, \mathrm{CH}_{\mathrm{b}}-\mathrm{O}\right), 3.49\left(\mathrm{~d}, 1 \mathrm{H}, \mathrm{CH}_{\mathrm{c}}-\mathrm{S}\right), 2.80\left(\mathrm{t}, 2 \mathrm{H}, \mathrm{C}_{7}-\mathrm{CH}_{2}\right), 2.24\left(\mathrm{t}, 2 \mathrm{H}, \mathrm{C}_{6}-\mathrm{CH}_{2}\right)$ ppm. Mass $\mathrm{m} / \mathrm{z}: 487,359,347,275,274,243,140,95$

5c IR: $v=3108(\mathrm{Al}-\mathrm{H}), 1630(\mathrm{C}=\mathrm{N}), 1078(\mathrm{C}-\mathrm{O}), 898(\mathrm{~N}-\mathrm{O}), 750(\mathrm{C}-\mathrm{Cl}), 685(\mathrm{C}-\mathrm{S}-\mathrm{C}) \mathrm{cm}^{-1}$. 'H NMR (DMSO-d6): $\delta=6.48-7.80(\mathrm{~m}$, $12 \mathrm{H}, \mathrm{Ar}-\mathrm{H}), 5.62\left(\mathrm{~s}, 1 \mathrm{H}, \mathrm{CH}_{3}\right), 4.52\left(\mathrm{~d}, 1 \mathrm{H}, \mathrm{CH}_{\mathrm{b}}-\mathrm{O}\right), 3.85\left(\mathrm{~s}, 3 \mathrm{H},-\mathrm{OCH}_{3}\right), 3.43(\mathrm{~d}, 1 \mathrm{H}, \mathrm{CHc}-\mathrm{S}), 2.78\left(\mathrm{t}, 2 \mathrm{H}, \mathrm{C}_{7}-\mathrm{CH}_{2}\right), 2.19(\mathrm{t}, 2 \mathrm{H}$, $\mathrm{C}_{6}-\mathrm{CH}_{2}$ ) ppm. Mass m/Z: 499, 371, 359, 287, 286,255, 140, 107

5d IR: $v=3102(\mathrm{Ar}-\mathrm{H}), 1628(\mathrm{C}=\mathrm{N}), 1071(\mathrm{C}-\mathrm{O}), 895(\mathrm{~N}-\mathrm{O}), 751(\mathrm{C}-\mathrm{Cl}), 680(\mathrm{C}-\mathrm{S}-\mathrm{C}) \mathrm{cm}^{-1}$. 'H NMR (DMSO-d $): \delta=6.25-7.45(\mathrm{~m}$, $13 \mathrm{H}, \mathrm{Ar}-\mathrm{H}), 5.60\left(\mathrm{~s}, 1 \mathrm{H}, \mathrm{CH}_{3}\right), 4.48\left(\mathrm{~d}, 1 \mathrm{H}, \mathrm{CH}_{\mathrm{b}}-\mathrm{O}\right), 3.48(\mathrm{~d}, 1 \mathrm{H}, \mathrm{CHc}-\mathrm{S}), 2.74\left(\mathrm{t}, 2 \mathrm{H}, \mathrm{C}_{7}-\mathrm{CH}_{2}\right), 2.21\left(\mathrm{t}, 2 \mathrm{H}, \mathrm{C}_{6}-\mathrm{CH}_{2}\right) \mathrm{ppm}$. Mass m/Z: 469, 341, 329, 257, 256, 225, 140, 77

$5 e \quad$ IR: $v=3085\left(\right.$ Ar-H), $1638(\mathrm{C}=\mathrm{N}), 1192(\mathrm{C}-\mathrm{F}), 1078(\mathrm{C}-\mathrm{O}), 910(\mathrm{~N}-\mathrm{O}), 750(\mathrm{C}-\mathrm{Cl}), 690(\mathrm{C}-\mathrm{S}-\mathrm{C}) \mathrm{cm}^{-1} .{ }^{1} \mathrm{H}$ NMR (DMSO-d $): \delta=$ $5.74\left(\mathrm{~s}, 1 \mathrm{H}, \mathrm{CH}_{3}\right), 4.50\left(\mathrm{~d}, 1 \mathrm{H}, \mathrm{CH}_{\mathrm{b}}-\mathrm{O}\right), 3.54\left(\mathrm{~d}, 1 \mathrm{H}, \mathrm{CH}_{\mathrm{c}}-\mathrm{S}\right), 2.85\left(\mathrm{t}, 2 \mathrm{H}, \mathrm{C}_{7}-\mathrm{CH}_{2}\right), 2.29\left(\mathrm{t}, 2 \mathrm{H}, \mathrm{C}_{6}-\mathrm{CH}_{2}\right)$ ppm. Mass m/z: 487, 363 , $359,291,290,259,124,111$

5f IR: $v=3078\left(\right.$ Ar-H), $1632(\mathrm{C}=\mathrm{N}), 1185(\mathrm{C}-\mathrm{F}), 1082(\mathrm{C}-\mathrm{O}), 902(\mathrm{~N}-\mathrm{O}), 690(\mathrm{C}-\mathrm{S}-\mathrm{C}), 687(\mathrm{C}-\mathrm{S}-\mathrm{C}) \mathrm{cm}^{-1}$. 'H NMR (DMSO-d $): \delta=$ 6.48-7.20 (m, 12H, Ar-H), $5.70\left(\mathrm{~s}, 1 \mathrm{H}, \mathrm{CH}_{3}\right), 4.48\left(\mathrm{~d}, 1 \mathrm{H}, \mathrm{CH}_{\mathrm{b}}-\mathrm{O}\right), 3.50\left(\mathrm{~d}, 1 \mathrm{H}, \mathrm{CH}_{\mathrm{c}}-\mathrm{S}\right), 2.74\left(\mathrm{t}, 2 \mathrm{H}, \mathrm{C}_{7}-\mathrm{CH}_{2}\right), 2.26\left(\mathrm{t}, 2 \mathrm{H}, \mathrm{C}_{6}-\mathrm{CH}_{2}\right)$ ppm. Mass in/Z: 471, 347, 343, 275, 274, 124, 95

5g IR: $v=3082(\mathrm{Ar}-\mathrm{H}), 1628(\mathrm{C}=\mathrm{N}), 1190(\mathrm{C}-\mathrm{F}), 1088(\mathrm{C}-\mathrm{O}), 899(\mathrm{~N}-\mathrm{O}), 690(\mathrm{C}-\mathrm{S}-\mathrm{C}) \mathrm{cm}^{-1}$. 'H NMR (DMSO-d 6$): \delta=6.52-7.48(\mathrm{~m}$, $12 \mathrm{H}, \mathrm{Al}-\mathrm{H}), 5.68\left(\mathrm{~s}, 1 \mathrm{H}, \mathrm{CH}_{3}\right), 4.56\left(\mathrm{~d}, 1 \mathrm{H}, \mathrm{CH}_{\mathrm{b}}-\mathrm{O}\right), 3.51\left(\mathrm{~d}, 1 \mathrm{H}, \mathrm{CH}_{-}-\mathrm{S}\right), 2.70\left(\mathrm{t}, 2 \mathrm{H}, \mathrm{C}_{7}-\mathrm{CH}_{2}\right), 2.19\left(\mathrm{t}, 2 \mathrm{H}, \mathrm{C}_{6}-\mathrm{CH}_{2}\right)$ ppm. Mass In/Z: 467, 343, 339, 286, 271, 255, 124, 107

5h IR: $v=3106(\mathrm{Ar}-\mathrm{H}), 1633(\mathrm{C}=\mathrm{N}), 1178(\mathrm{C}-\mathrm{F}), 1082(\mathrm{C}-\mathrm{O}), 907(\mathrm{~N}-\mathrm{O}), 688(\mathrm{C}-\mathrm{S}-\mathrm{C}) \mathrm{cm}^{-1}$. 'H NMR (DMSO-d 6$) ; \delta=6.64-7.75(\mathrm{~m}$, 13H, Ar-H), $5.63\left(\mathrm{~s}, 1 \mathrm{H}, \mathrm{CH}_{3}\right), 4.58\left(\mathrm{~d}, 1 \mathrm{H}, \mathrm{CH}_{\mathrm{b}}-\mathrm{O}\right), 3.48\left(\mathrm{~d}, 1 \mathrm{H}, \mathrm{CH}_{-}-\mathrm{S}\right), 2.74\left(\mathrm{t}, 2 \mathrm{H}, \mathrm{C}_{7}-\mathrm{CH}_{2}\right), 2.28\left(\mathrm{t}, 2 \mathrm{H}, \mathrm{C}_{6}-\mathrm{CH}_{2}\right)$ ppm. Mass $\mathrm{m} / \mathrm{z}: 453,329,325,257,256,225,124,77$ 
yl) is an ambivalent electrophile where the carbonyl carbon is hard and $\beta$-carbon is the soft centre. The conjugation of the double bond with the phenyl ring reduces the extent of its conjugation with the carbonyl group. Where as in case of hydroxylamine the nucleophilicity of $\mathrm{N}$ is increased due to adjacent oxygen atom, thus make it a harder nucleophile ( $\alpha$ effect). Hence the attack of $\mathrm{NH}_{2}$ at carbonyl occurs first forming the isoxazole derivatives $5 \mathbf{a l}$ h and not its alternate structure. The ${ }^{1} \mathrm{H}$ NMR spectrum of 5 a was in accordance with the proposed structure. The diagnostic methine products $(\mathrm{O}-\mathrm{CH}-\mathrm{CH}-\mathrm{S})$ attached to $\mathrm{O}$ and $\mathrm{S}$ appeared as clear doublet $(J=3.80 \mathrm{~Hz})$ at around $\delta 4.60$ and $\delta 3.52$ respectively confirming the condensation reaction with hydroxylamine hydrochloride. Finally conclusive evidence has been gathered from the mass spectrum of all final compounds. The approach of generating the targeted systems has also been carried under themnal cyclisation conditions with a view to compare the effectiveness of microwave irradiation. Conventional heating gave product but in low yields compare to microwave irradiation. Physical and analytical data are presented in (Table 1) and spectral data are given in (Table 2).

\section{Experimental Section}

General Procedures. All the reactions were carried out in a domestic microwave oven (Kenstar, Model No. OM-26 EGO, Power $1200 \mathrm{~W}$ ). Melting points were determined in open capillaries. Reactions were monitored by thin layer chromatography using silica gel-G as adsorbent using ethyl acetate: benzene (1:9) as eluent. IR spectra ( $\mathrm{KBr}$ pellets) were recorded on Perkin-Elmer 1800 (FTIR) Spectrometer. 'H NMR spectra (DMSO-d $\mathrm{d}_{6}$ ) were taken a Bruker DRX spectrometer ( $300 \mathrm{MHz}$ FT NMR) using TMS as intemal standard and chemical shift are expressed in $\delta$ ppm. Mass spectra were taken on Jeol sx-102/PA-6000 (EI) spectrometer. Compounds 1ald were prepared according to literature reported. ${ }^{22}$

Synthesis of 4-Aryl-3,4,5,6-tetrahydrobenzo(h)quinazoline-2(1H) thiones (2a-d).

Conventional Method: A mixture of 2-arylidenetetralin1-one 1n-d $(0.02 \mathrm{~mol})$ and thiourea $(0.02 \mathrm{~mol})$ and $\mathrm{KOH} 2 \mathrm{~g}$ was taken in ethanol and refluxed for 4-6 hr. The reaction was monitored on TLC. After the completion of the reaction, it was cooled and the resulting solid was filtered, dried and recrystallized from ethanol to get compound $2 \mathrm{a}-\mathrm{d}$.

Microwave Method: A mixture of 2-arylidenetetralin-1one $1 \mathrm{a}-\mathrm{d}(0.02 \mathrm{~mol})$ and thiourea $(0.02 \mathrm{~mol})$ in ethanolic $\mathrm{KOH}(1 \mathrm{~g} \mathrm{KOH}$ in $25 \mathrm{~mL}$ ethanol) was irradiated under MWI for $5.30 \mathrm{~min}$. with a time interval of 10 seconds. The volume of the reaction mixture was reduced to half and kept ovemight. The solid thus separated as shining needles was filtered and washed with aqueous ethanol to give 2 a-d.

Synthesis of 5-Aryl-2,3,6,7-tetrahydro-3-0xo-5H-thiazolo-[2,3-b]benzo(h)quinazolines (3a-d).

Conventional Method: A mixture of thiones 2a-d $(0.01$ $\mathrm{mol})$, chloroacetic acid $(0.01 \mathrm{~mol})$, anhydrous sodium acetate $(0.01 \mathrm{~mol})$, and acetic anhydride $(2 \mathrm{~mL})$ was taken in glacial acetic acid and refluxed for 3-5 hr. After cooling the reaction mixture, it was poured into ice cool water. The solid product was filtered, dried and recrystallized from acetic acid to get (3a-d).

Microwave Method: A mixture of thiones 2a-d $(0.01$ mol), chloroacetic acid $(0.01 \mathrm{~mol})$, anhydrous sodium acetate $(0.01 \mathrm{~mol})$, glacial acetic acid $(15 \mathrm{~mL})$ and acetic anhydride $(2 \mathrm{~mL})$ was irradiated under microwave irradiation for 2 min. using funnel as a loose top. The reaction mixture was cooled and poured into ice-cold water. The solid thus separated was filtered, washed with water and finally crystallized from glacial acetic acid to give 3 a-d.

Synthesis of 5-Aryl-2arylidine-2,3,6,7-tetrahydro-3oxo-5H-thiazolo[2,3-b]benzo[h]quinazolines (4a-h).

Conventional Method: A mixture of 3a-d $(0.01 \mathrm{~mol})$ and appropriate aromatic aldehydes $(0.01 \mathrm{~mol})$ was taken in glacial acetic acid. Anhydrous sodium acetate $(0.02 \mathrm{~mol})$ was added to it and refluxed for $3 \mathrm{hr}$. The reaction mixture was allowed to attain room temperature and treated with cold water. The solid thus separated was filtered, washed with water and recrystallized from glacial acetic acid to furnish compounds 4a-h.

Microwave Method: Compounds 3a-d (0.01 mol) was suspended in minimum quantity of ethanol. To this appropriate aromatic aldehydes $(0.01 \mathrm{~mol})$, anhydrous sodium acetate $(0.02 \mathrm{~mol})$ and glacial acetic acid $(10 \mathrm{~mL})$ were added and irradiated for the $1.50-2.50 \mathrm{~min}$. The reaction mixture was cooled at room temperature and then poured into ice cold water. The separated solid was filtered, washed with water and crystallized from glacial acetic acid to give $\mathbf{4 a - h}$.

Synthesis of 7,11-Diphenyl5,6a,7,11,11a,13a-hexahydro6H-benzo[h]isoxazolo $\left[3^{\prime}, 4^{\prime}, 4,5\right][1,3]$ thiazolo[2,3-b]quinazolines (5a-h).

Conventional Method: A mixture of 4a-h $(0.01 \mathrm{~mol})$ and hydroxylamine hydrochloride $(0.012 \mathrm{~mol})$ was taken in absolute ethanol. To it solution of sodium acetate $(0.012$ mol) in acetic acid $(5 \mathrm{~mL})$ was added slowly. It was refluxed for $10 \mathrm{~h}$. The reaction mixture was kept for overnight and then solution was poured into water. The resulting solid was filtered, dried and recrystallized from acetic acid to get product 5a-h.

Microwave Method: Solution of sodium acetate $(0.012$ mol) in acetic acid $(5 \mathrm{~mL})$ was added to a mixture of $4 \mathbf{a}-\mathbf{h}$ $(0.01 \mathrm{~mol})$ and hydroxylamine hydrochloride $(0.012 \mathrm{~mol})$ in absolute ethanol. The reaction mixture was irradiated for $6-8$ $\mathrm{min}$. under microwave and kept overnight, solution was poured into water. The solid thus obtained was crystallized from acetic acid to give 5a-h.

Biological Screening. In view of antimicrobial activities of some quinazoline derivatives, it was of interest to incorporate this moiety into some heterocyclic molecules with the hope that the resulting compounds might exhibit enhanced activity compared to the parent quinazoline derivatives. The products and their parent substances were tested in vitro for their antibacterial activity against Pseudomonas aenuginosa, Proteus mirabilis, Klebsiella pneumoniae, Bacillus stbtilis, and antifungal activity against Aspergillus fumigatus, Candida 
Table 3. The antimicrobial activitics of some synthesized compounds

\begin{tabular}{|c|c|c|c|c|c|c|}
\hline \multirow[b]{2}{*}{ Compd. } & \multicolumn{4}{|c|}{ Antibacterial Activity (250 ppm) } & \multicolumn{2}{|c|}{ Antifungal Activity (250 ppm) } \\
\hline & $\begin{array}{c}\text { Pseudomonas } \\
\text { aeruginosa }\end{array}$ & $\begin{array}{l}\text { Protents } \\
\text { minabilis }\end{array}$ & $\begin{array}{c}\text { Klebsiclla } \\
\text { preumoniae }\end{array}$ & $\begin{array}{l}\text { Bacillus } \\
\text { subtilis }\end{array}$ & $\begin{array}{l}\text { Aspergillus } \\
\text { fumigatis }\end{array}$ & $\begin{array}{l}\text { Candida } \\
\text { albicans }\end{array}$ \\
\hline $\mathbf{3 b}$ & - & + & - & + & + & - \\
\hline $3 c$ & + & + & - & - & + & + \\
\hline 3d & - & - & + & + & + & - \\
\hline $\mathbf{5 b}$ & - & - & + & - & - & + \\
\hline $5 c$ & $+1+$ & ++ & $+1+$ & $+1+$ & + & - \\
\hline $5 \mathbf{d}$ & - & $+1+$ & + & ++ & - & $+1+$ \\
\hline $5 \mathbf{e}$ & $+1+$ & - & $+1+$ & - & $+1+$ & - \\
\hline $\mathbf{5 f}$ & ++ & + & - & + & + & + \\
\hline $5 g$ & $+1+$ & $+1+$ & $+H$ & - & - & $+1+$ \\
\hline Ciprofloxacin & $+1+$ & +1 & +1 & $+1+$ & - & - \\
\hline Amphotericin B & - & - & - & - & $+1+$ & $+1+$ \\
\hline
\end{tabular}

-: No activity; +: moderate activity (inhibition zone: $5-10 \mathrm{~mm}$ ); ++ : strong activity (inhibition zone: $11-15 \mathrm{~mm}$ ); +1+: very strong activity (inhibition zone: $16-20 \mathrm{~mm}$ ).

albicans, by the cup or well ${ }^{23}$ method using DMF as a solvent at the concentration of $250 \mathrm{ppm}$. Commercial antibacterial Ciprofloxacin and antifungal Amphotericin B were also screened under similar conditions for comparison. The diameter of inhibition zones $(\mathrm{mm})$ were measured and recorded. The results have been tabulated in the form of inhibition zones in (Table 3 ). The resulting compounds showed better activity against tested organisms. In general, the introduction of thiazolidinone and isoxazole heterocyclic system in the quinazoline nucleus results in the enhancement of the activity of the compounds.

\section{Conclusion}

In conclusion an efficient synthesis of quinazoline derivatives carrying potential pharmacophores like thiazolidinone and isoxazole have been prepared in an environmentally benign microwave protocol. The yields of the products formed under MWI were high in comparison to classical method (Table 1) and time required for completion of these reactions was also less in comparison to classical method. By visualizing the antimicrobial data (Table 3 ) it could be observed that most of the final compounds exhibited moderate to strong activities against all the tested organisms.

Acknowledgements. The authors are thankful to Head, Department of Chemistry, M. L Sukhadia University, Udaipur (Raj.) for providing laboratory facilities and Dept. of Biotechnology (M. L. Sukhadia University, Udaipur) for antimicrobial screening. Authors are also thankful to the Director, RSIC, CDRI, Lucknow for spectral and analytical studies. One of the authors (SM) is also thankful to CSIR-New Delhi for providing necessary financial assistance.

\section{References}

1. Mazzoni, O.; Bosco, A. M.; Grieco, P.; Novellino, E.; Bertamino,
A.; Borreli, F,; Capasso, R.; Diumo, M. V. Chem. Biol. Drugs Des. 2006, 67, 432 .

2. Diurno, M. V.; Piscopo, E.; Mazzoni, O.; Calignano, A. Boll. Soc. Ifal. Biol. Sper. 1991, 67, 1067.

3. Vigorila, M. G.; Otlana, R,; Monforti, F.; Maccari, R.; Trovalo, A. Monforte, M. T.; Taviano, M. F. Bioorg. Med. Chem. Lett, 2001, 11,2791 .

4. Siddiqui, I. R.; Singh, P. K.; Singh, J. J. Agric Food Cheni. 2005. 5,7062 .

5. Ottana, R.; Maccari, R.; Barreca, M. L.; Bruno, G; Rotondo, A.; Rossi, A.; Chiricosta, G.; Di Paolo, R.; Sautebin, L.; Cuzzoctea, S.; Viqorita, M. G. Bioorg. Med. Chem. 2005, 13,4243.

6. Previtera, T.; Vigorila, M. G; Fenech, G; Zappaala, C.; Giordano, A.; Monforte, M. T.; Forestieri, A. M. Farmaco. 1994, 49, 33.

7. Rawal, R. K.; Solomon, V. R.; Prabhakar, Y. S.; Katti, S. B.; De Clercq, E. Comb. Chent. High throughput Screen 2005, 8, 439.

8. Gududuru, V; Hurh, E.; Dalion, J. T.; Miller, D. D. J. Med. Chem. $2005,48,2584$

9. Roy, R. V; Desai, K. R. Int. J. Chem, Sci, 2005, 3,529.

10. Dandia, A.; Singh, R.; Arya, K. Phosphorous, Stlphir \& Silicon and Related Elements 2004, 179, 551 .

11. Kai, H.; Masumoto, H.; Hattori, N.; Takasa, A.; Fujiwana. T.; Sugimolo, H. Bioorg. Med. Chem. Lett, 2001, 11, 1997.

12. Rampal, O.; Handa, R. N.; Pujari, H. K. Indian J. Chem, 1994, $33 B, 629$.

13. Jhone, S. In Progress in Drug Research; Juker, E., Ed.; Birkauser Verlag: Basel, 1982; Vol. 26, p 259.

14. Brown, D. J. The Chemistry of Heterocyclic Compounds; J. Wiley \& Sons: New York, 1996.

15. Metwally, M.; Etman, H.; Keshl, E.; Fekry, A. Phosphorzis, Sulfin; and Silicon, and the Related Elements. 2006, 181, 1039.

16. Ahmed, M. S. M.; Kobayashi, K.; Mori, A. Org. Lett. 2005, 7, 4487.

17. Seyas, J. A.; Vazquez-Tato, M. P.; Marting, M. M. Tetrahedron Lett. $2000,41,2215$.

18. Mahmood, M. R. Indian J. Chem. 1990, 29B, 830

19. Theoclitou, M. E.; Robinson, L. A. Tetrahedron Lett, $2002,43,3907$.

20. Zhao, Z,; Leister, W. H.; Strauss, K. A.; Wisnoski, D. D.; Lindsley, C. W. Tetrahedron Lett. 2003, 44, 1123.

21. Mont, N.; Teixidó, J.; Borell, J. I.; Kappe, C. O. Tetrahedron Lett. $2003,44,5385$.

22. Mahmood, M. R. Indian J. Chem. 1990, 29B, 830.

23. Collee, G J.; Fraser, G. A.; Mamion, P. B.; Suninous, A. Practical Medical Microbiolog; $14^{\text {th }}$ ed.; Churchil! Livingstone, Edinburgh: 1996; Vol. II, p 163. 